Memorias del VII Encuentro Nacional de Experiencias en la Enseñanza de la Biología y la Educación Ambiental y II Congreso Nacional de Investigación en la Enseñanza de la Biología

\title{
APROXIMACIÓN A LAS CONCEPCIONES ACERCA DE CIRCULACIÓN DE ESTUDIANTES DE SÉPTIMO GRADO DE UNA INSTITUCIÓN EDUCATIVA OFICIAL DE LA CIUDAD DE NEIVA
}

\section{APPROXIMATION TO THE CONCEPTIONS BRINGS OVER OF STUDENTS' TRAFFIC OF THE UNA'S SEVENTH DEGREE EDUCATIONAL OFFICIAL INSTITUTION OF NEIVA'S CITY}

\author{
Susana Rodríguez Rodríguez ${ }^{1}$ \\ Elías Francisco Amórtegui Cedeño
}

\section{Resumen}

A continuación se presentan los resultados preliminares del desarrollo de práctica pedagógica I al interior del Programa de Licenciatura en Educación Básica con Énfasis en Ciencias Naturales y Educación Ambiental de la Universidad Surcolombiana (Neiva, Huila-Colombia) llevada a cabo en una Institución Educativa Oficial. El grupo de trabajo está conformado por estudiantes de séptimo grado cuyas edades oscilan entre 11 y 16 años. Esta ponencia se refiere particularmente a la planificación para la práctica pedagógica 1 y además mostramos una aproximación a las concepciones acerca del concepto circulación que tienen los estudiantes. La metodología está enmarcada en una perspectiva cualitativa, empleando el análisis de contenido y haciendo uso del cuestionario. Los resultados permitieron reconocer tres tendencias tanto para el concepto general de circulación, como para lo específico de la circulación en plantas y en animales/seres humanos. Finalmente, se muestran algunas implicaciones en la enseñanza-aprendizaje de las Ciencias Naturales y se proyectan algunas posibles estrategias para favorecer al aprendizaje del concepto.

\section{Abstract}

Here are preliminary results of the development of teaching practice I into the Bachelor of Education Program with Emphasis on Basic Science and Environmental Education at the Universidad Surcolombiana (Neiva, Huila, Colombia) held in an Educational Institution Officer. The working group is

\footnotetext{
${ }^{1}$ Estudiante de noveno semestre del Programa de Licenciatura en Educación Básica con Énfasis en Ciencias Naturales y Educación Ambiental. Universidad Surcolombiana chana0528@hotmail.com

2 Docente de Planta Tiempo Completo. Programa de Licenciatura en Educación Básica con Énfasis en Ciencias Naturales y Educación Ambiental. Universidad Surcolombiana. Magister en Educación. elias.amortegui@usco.edu.co

Semillero de Investigación ENCINA Grupo de Investigación Interinstitucional Conocimiento Profesional del Profesor de Ciencias
} 
Memorias del VII Encuentro Nacional de Experiencias en la Enseñanza de la Biologia y la Educación Ambiental y II Congreso Nacional de Investigación en la Enseñanza de La Biología

comprised of seventh-grade students aged between 11 and 16 years. In the case of this paper we refer particularly to planning for one pedagogical practice and also show an approximation to the conceptions of circulation concept for students. The methodology is framed in a qualitative perspective, using content analysis and using the questionnaire. The results showed three trends for both the general concept of movement, as to the specifics of the circulation in plants and animals I humans. Finally, we show some implications for teaching and learning of Science and project some possible strategies to promote the learning of the concept.

Palabras clave: Circulación, Concepciones, Planificación, Ciencias Naturales y Educación Ambiental.

Keywords: Circulation, Concepts, Planning, Science and Environmental Education.

\section{Introducción}

El Programa de Licenciatura en Educación Básica con Énfasis en Ciencias Naturales y Educación Ambiental, es un programa de formación inicial de docentes adscrito a la Facultad de Educación de la Universidad Surcolombiana (Neiva-Huila); éste, consta de nueve semestres y cuatro ámbitos de formación (Biología, Química, Física y Didáctica) que componen el núcleo común dentro del componente básico específico en el plan de estudios. Al interior del programa, se realizan dos prácticas pedagógicas llevadas a cabo al interior de octavo y noveno semestre respectivamente, realizándose la Práctica Pedagógica I en el nivel de la básica secundaria en una institución educativa de cualquier municipio del Departamento del Huila, en el área de Ciencias Naturales y Educación Ambiental; mientras que la Práctica Pedagógica II se lleva a cabo en la Educación Media, particularmente en el área de Química o Física.

En este caso, ambas prácticas pedagógicas tienen una duración de dieciocho semanas, de las cuales la segunda corresponde al proceso de observación del grupo de estudiantes por parte del futuro docente, en donde examina la dinámica de la clase teniendo en cuenta aspectos como los gustos, intereses, experiencias, ideas previas, dificultades de aprendizaje de los estudiantes, entre otros; las siguientes dieciséis semanas son de intervención directa junto con el estudiantado por parte del futuro docente, en donde pone en juego los conocimientos adquiridos en su formación inicial.

Para el caso de la primer semana, el practicante futuro docente realiza una previa planificación junto con el coordinador de práctica pedagógica del programa, sus compañeros y un docente asesor quien cuenta con amplia formación en investigación educativa, pedagógica y didáctica, teniendo en cuenta aspectos 
Memorias del VII Encuentro Nacional de Experiencias en la Enseñanza de la Biologia y la Educación Ambiental y II Congreso Nacional de Investigación en la Enseñanza de La Biología

tales como: el modelo didáctico que orienta la práctica pedagógica, los requerimientos y requisitos de la institución educativa al igual que los contenidos dentro del plan de estudios del curso a intervenir, las finalidades de la Práctica Pedagógica, las posibles estrategias a implementar de acuerdo a las bases teórico-pedagógicas que se tienen, Criterios y mecanismos de Evaluación, actividades CTS-A, uso de TICs, entre otros.

Para el caso de Colombia, las directrices planteadas por el Ministerio de Educación Nacional, contemplan dentro de los Estándares y Competencias para los grados de sexto a séptimo, las funciones de los seres vivos a partir de las relaciones entre diferentes sistemas de órganos, es por esto que para el caso de esta ponencia nos referimos al concepto de circulación.

Los seres vivos obtienen de su medio ambiente, las sustancias y los gases necesarios para realizar todos sus procesos internos, y al tiempo eliminan las sustancias de desecho, pues su acumulación puede producir la muerte. Por lo tanto la Circulación es el medio de transporte de estas sustancias entre las diferentes células del cuerpo de cualquier organismo. Existiendo así diferentes tipos de circulación según el organismo del que se trate, de esta manera los unicelulares manifiestan transporte celular y los pluricelulares tales como los invertebrados (con algunas excepciones) y los vertebrados, presentan sistemas circulatorios integrados por un órgano motor, un liquido circulante y vasos conductores, en sistemas sean abierto o cerrado.

Para el caso de esta ponencia, definimos las concepciones tal como plantea Amórtegui (2011) como sistemas de ideas que tienen los sujetos que pueden evolucionar a través de un proceso de reestructuración que puede o no ser consciente, basado en la interacción con otras ideas y experiencias de los sujetos, teniendo en cuenta que las concepciones originadas en un contexto particular que provocan un aumento en su grado de complejidad no se transfieren de manera automática y mecánica a otros contextos y problemas de la misma clase, pero si pueden influir en ellos, están fuertemente arraigadas en la medida que son coherentes, flexibles y funcionales $y$, posibilitan explicaciones causales a fenómenos físicos y al igual que las rutinas, son resistentes al cambio y consecuentemente, pueden constituir obstáculos para la transformación. Para el caso de esta ponencia, cabe resaltar que se refiere exclusivamente a las respuestas textuales de los estudiantes a partir de la aplicación de un cuestionario.

Específicamente en el campo de la enseñanza-aprendizaje de la Biología, las concepciones son fundamentales debido a que los estudiantes tienen explicaciones sobre los fenómenos biológicos que difieren de los aceptados desde 
Bio-grafía Escritos sobre la Biología y su Enseñanza.

Edición Extra-Ordinaria. ISSN 2027-1034 P. p320-328

Memorias del VII Encuentro Nacional de Experiencias en la Enseñanza de la

Biología y la Educación Ambiental y II Congreso Nacional de Investigación en

la Enseñanza de la Biología

el punto de vista científico, formadas de forma espontánea como consecuencia de sus percepciones sobre los fenómenos naturales (Banet, 2000).

Con relación a los antecedentes, cabe destacar que por espacio no los presentamos, sin embargo para el caso del Departamento del Huila y la Ciudad de Neiva no existen trabajos sobre la aproximación a las concepciones que tienen los estudiantes sobre el concepto de circulación, ni sobre las implicaciones de estas en la enseñanza-aprendizaje de las Ciencias Naturales.

\section{Metodología}

El estudio se llevó a cabo desde un enfoque cualitativo, empleando el método de análisis de contenido en el proceso de sistematización, y usando como herramienta de recolección de información el cuestionario, el cual consistió en 6 preguntas abiertas en donde los niños debian argumentar sus respuestas a través de escritos y dibujos. Cabe destacar que el estudio fue de tipo transversal y por ende corresponde exclusivamente al diagnostico de las concepciones (se espera en otros eventos mostrar el seguimiento de dichas concepciones) El grupo objeto de estudio consistió en 33 estudiantes entre 11 y 16 años de edad, pertenecientes en su mayoría a los estratos socioeconómicos 1 y 2 que cursan séptimo grado en una Institución Educativa Oficial de la ciudad de Neiva. Particularmente la ponencia se refiere a las respuestas de los estudiantes frente a las preguntas: "¿para ti qué es cirulación?, ¿qué circula en una planta?, ¿qué circula en los animales y en tu cuerpo?".

\section{Resultados y discusión}

Los hallazgos permitieron establecer tres tendencias para cada una de las categorías de estudio: circulación, circulación en plantas y circulación en animales/seres humanos. A continuación presentamos las características de cada una, así como su frecuencia y en algunos casos mostramos evidencias textuales de las respuestas de los estudiantes.

\section{Circulación}

Como proceso: Esta es la tendencia mayoritaria (14 estudiantes) en la que los educandos conciben la circulación como un Proceso que se lleva tanto al interior como en el exterior de los seres vivos.

E13.C1.1: "Circulación es un proceso que se lleva a cabo ya sea dentro o fuera de los seres vivos". 


\begin{abstract}
Bio-grafía Escritos sobre la Biología y su Enseñanza.
Edición Extra-Ordinaria. ISSN 2027-1034 P. p 320-328

Memorias del VII Encuentro Nacional de Experiencias en la Enseñanza de la Biología y la Educación Ambiental y II Congreso Nacional de Investigación en la Enseñanza de La Biología
\end{abstract}

E22.C1.1: "La circulación es un proceso que se cumple tanto adentro como afuera de todo ser vivo"

Lo anterior es fundamental en la medida en la que los estudiantes reconocen que existe un componente al interior de los seres vivos y en tal forma hace parte de la dinámica de su funcionamiento a pesar de que no especifican como tal las estructuras o fisiología de la circulación. Esto muestra que estos estudiantes se centran en una vision sistemica desde la perspectiva de Capra (1998) en la que los sistemas vivos son considerados como sistemas abiertos en constante dinamica en la que existe un medio interno y un medio externo en el cual existe un flujo de materia y energía. De manera indirecta como se pudo observar en las clases, los estudiantes relacionan la circulación al interior de los seres vivos con los fenómenos sociales y de movilidad en las grandes ciudades en los cuales ellos están inmersos; en tal sentido lo anterior está estableciendo una relación entre el conocimiento científico y el conocimiento cotidiano de los estudiantes en aras de en un futuro construir un conocimiento escolar contextualizado y epistemológicamente diferenciado de los dos anteriores (Arnay, 1997: García, 1998).

Como movimiento: en este caso la tendencia es considerable ya que son 12 estudiantes los que conciben a la circulación como un Movimiento de productos o nutrientes que adquiere dichos seres vivos a partir del ecosistema que les rodea.

E2.C1.1: "La circulación es el movimiento de nutrientes que adquiere todo ser vivo a partir del ecosistema que les rodea".

E29.C1.1: "Circulación es el movimiento de productos o nutrientes que los seres vivos toman a partir del ecosistema que les rodea".

Es fundamental esta propuesta de los estudiantes pues a diferencia de la anterior, reconocen no solo el proceso sino aquellos elementos que participan dentro de la circulación y específicamente a los nutrientes, sin embargo es de destacar que no reconocen otros elementos que circulan, como el oxigeno, entre otros. A diferencia de la tendencia anterior, estos estudiantes manifiestan una visión centrada en el medio interno de los organismos sin tener en cuenta las características y las relaciones de este con el medio externo.

Como transporte: con relación a esta tendencia fueron tan solo 7 estudiantes que se corresponden al concebir la circulación como un Transporte e intercambio de sustancias o fluidos de un lugar a otro ya sea dentro o fuera de un cuerpo.

E17.C1.1: "Para mí la circulación es el intercambio o el transporte de fluidos de un lugar a otro ya sea dentro o fuera de un cuerpo". 


\begin{abstract}
Bio-grafía Escritos sobre la Biología y su Enseñanza.
Edición Extra-Ordinaria. ISSN 2027-1034 P. p320-328

Memorias del VII Encuentro Nacional de Experiencias en la Enseñanza de la Biología y la Educación Ambiental y II Congreso Nacional de Investigación en la Enseñanza de la Biología
\end{abstract}

E24.C1.1: "Circulación es el transporte de nutrientes de un lugar a otro que se lleva a cabo tanto adentro como afuera del organismo".

Esta idea corresponde de manera muy similar a la primera agrupación, sin embargo contempla que dicho transporte se realiza dentro del organismo como fuera de él y principalmente con base en nutrientes, lo cual es importante en aras de reconocer que los organismos requieren de éstos para el mantenimiento, la regulación, el crecimiento y el desarrollo.

\title{
Circulación en plantas
}

En este caso la tendencia considerada mayoritaria con 13 estudiantes fue el concepto relacionado con La Estructura y Contenido de la planta, puesto que dichos sujetos se refieren exclusivamente a los medios en los cuales circulan las sustancias, para este caso el xilema, el floema, la fotosíntesis, la raíz, el tallo y las hojas quienes se encargan de circular sales minerales, agua y luz solar.

E6.C1.1: "En el interior de una planta circula sales minerales, agua y luz solar $\underline{\text { a }}$ través del xilema, floema y tallo".

E15.C1.1: "En el interior de una planta circula la fotosíntesis a través del xilema y floema".

De acuerdo a lo anterior los estudiantes relacionan sus preconceptos inmediatamente con las partes de las plantas y con lo que verdaderamente fluye dentro de ella, lo cual nos quiere decir que a pesar de ubicar con precisión el concepto en la idea competente no tienen bien claro la fisiología de cada estructura que compone a la planta; y esto se debe principalmente a la falta de atención o falta de capacidad de lograr un aprendizaje significativo en años anteriores.

Ahora bien, la tendencia referida a la estructura son 12 estudiantes quienes conciernen que en el interior de las plantas circulan estructuras tales como el xilema, floema, raíz, tallo, frutos, flores y hojas.

E1.C1.1: "en el interior de una planta circula el tallo, el fruto, el xilema y la raíz".

E33.C1.1: "En una planta circula la semilla, las flores, el fruto, el xilema y las hojas".

Esta idea presenta gran similitud a la anterior puesto que los estudiantes consideran según su experiencia adquirida anteriormente que dentro de las plantas circula o se transportan lo que se observa en el exterior, es decir que las 
Memorias del VII Encuentro Nacional de Experiencias en la Enseñanza de la Biologia y la Educación Ambiental y II Congreso Nacional de Investigación en la Enseñanza de la Biología

estructuras externas siguen siendo relacionadas con la vital circulación; no obstante, cabe destacar una palabra que manifiestan constantemente los estudiantes aunque no tengan bien claro su función y ubicación, tal es el caso del concepto xilema, término que aterrizan inmediatamente los estudiantes al mencionarles circulación en las plantas pero aclaran en sus respuestas que es una estructura que fluye dentro de la misma. Esto se debe principalmente a la falta de interés por retroalimentar sus conocimientos constantemente. Lo anterior esta relacionado con el hecho de que muchos estudiantes mantienen conceptos de circulacion desde una perspectiva más estructural que funcional (Perez, 1993). $Y$ por ultimo en lo relacionado con el contenido son tan solo 7 estudiantes quienes conciben que en el interior de una planta circula sales minerales, nutrientes y agua.

E5.C1.1: "En el interior de una planta circula sales minerales y agua".

E24.C1.1: "En el interior de una planta circula agua y aire".

\section{Circulación en animales/seres humanos}

En cuanto a esta categoría se manifestaron 3 tipos de tendencias, es decir estudiantes que se corresponden expresando la parte estructural como circulación en los animales/seres humanos; otros, la parte interna (contenido) y por último la parte estructural e interna.

Ahora bien la tendencia considerada mayoritaria con 16 estudiantes es aquella en donde relacionan la parte interna de todo animal/ser humano como su circulación vital, es decir el oxigeno, la sangre, las neuronas y los nutrientes.

E7.C1.1: "En el interior de un animal/ser humano circula la sangre, el oxigeno y las neuronas".

E20.C1.1: "En un animal/ser humano circula los nutrientes, la sangre y el oxigeno".

Lo anterior, es fundamental en aras de sobrepasar la concepción de los estudiantes en la cual circula exclusivamente oxígeno a través del sistema viviente, sino también nutrientes; lo cual esta relacionado con una visión precientifica tal como plantea Mosquera (2012) en la cual la circulación es entendida como punto central en la nutrición a través de procesos tales como la digestión, la absorción, la ingestión, entre otros.

Seguidamente de la mayoritaria es posible hallar una tendencia considerable con 13 estudiantes que se corresponden al expresar un concepto relacionado con la 
Bio-grafía Escritos sobre la Biología y su Enseñanza.

Edición Extra-Ordinaria. ISSN 2027-1034 P. p320-328

Memorias del VII Encuentro Nacional de Experiencias en la Enseñanza de la

Biologia y la Educación Ambiental y II Congreso Nacional de Investigación en

la Enseñanza de la Biología

parte tanto estructural como interna del animal/ser humano, es decir, en dichos seres vivos circula los músculos, las proteínas, los alimentos, el agua y la sangre.

E4.C1.1: "En los animales y seres humanos les circula los músculos, las proteínas, los alimentos y el agua".

E31.C1.1: "En el interior de un animal y un ser humano circula la sangre, los músculos y los alimentos".

Finalmente, se encontró la tendencia correspondiente a la estructura del ser humano y animal como su circulación vital, es decir, en el interior de dichos seres vivos circulan los huesos, intestinos, el cerebro, los músculos y los tejidos.

E1.C1.1: "A través de un animal/ser humano circulan los huesos, intestinos, músculos, hígado, los tejidos epiteliales y el cerebro".

E24.C1.1: "A través de un animal o un ser humano circulan los huesos, los músculos, el cerebro y los tendones".

Consideramos importante favorecer estrategias de enseñanza que permitan abordar tanto contenidos conceptuales, como procedimentales y actitudinales, en este sentido favorecer la integración entre los procesos de digestión, circulación y respiración a través de la interpretación de diagramas e imágenes y la generación de actitudes críticas frente a aspectos tales como las dietas alimenticias (Jiménez, 2003).

Finalmente es de destacar que ninguna de las tendencias muestran una fuerte relación con los procesos de digestión y respiración los cuales desde la perspectiva de Nuñez y Banet (1996) permiten comprender de mejor manera el funcionamiento del cuerpo humano, y desde allí la importancia que los maestros elaboren propuestas didácticas en las que contemplen la circulación en el marco del proceso de nutrición.

\section{Conclusiones}

Dado que nuestro trabajo corresponde a una aproximación descriptiva, consideramos que la mayoría de estudiantes se acercan a ideas bastantes complejas y enriquecedoras acerca del concepto circulación, en tanto lo reconocen desde tres perspectivas, las estructuras, el funcionamiento y el contenido relacionado con los procesos de circulación. En el primero se refieren a estructuras tales como el xilema y el floema para el caso de las plantas, en el segundo dan cuenta de ideas sobre transporte y movimiento y por último se 
Bio-grafía Escritos sobre la Biología y su Enseñanza.

Edición Extra-Ordinaria. ISSN 2027-1034 P. p 320-328

Memorias del VII Encuentro Nacional de Experiencias en la Enseñanza de la Biología y la Educación Ambiental y II Congreso Nacional de Investigación en la Enseñanza de la Biología

refieren a los nutrientes, sangre y oxígeno como contenidos que circulan en los sistemas vivientes.

\section{Bibliografía}

- Amórtegui, E (2011). Concepciones sobre prácticas de campo y su relación con el conocimiento profesional del profesor, de futuros docentes de Biología de la Universidad Pedagógica Nacional. Tesis para optar al título de Magíster en Educación. Bogotá DC: Universidad Pedagógica Nacional

- Arnay, J. (1997). Reflexiones para un debate sobre la construcción del conocimiento en la escuela: Hacia una cultura científica escolar. En: Rodrigo, M.J. y Arnay, J. (comps). La construcción del conocimiento escolar. Barcelona: Piados. pp. 35-58.

- Banet, E (2000). Enseñanza aprendizaje del conocimiento Biológico. En: Perales, F \& Cañal, P (2000). Didáctica de las ciencias experimentales. Madrid: Acoy.

- García, E. (1998). La naturaleza del conocimiento escolar: ¿transición de lo cotidiano a lo científico o de lo simple a lo complejo? En: Rodrigo, M.J. y Arnay, J. (comps). La construcción del conocimiento escolar. Barcelona: Paidós. pp. 59-80.

- Jiménez, M (2003). La enseñanza y el aprendizaje de la Biología. En: Jiménez, M et al (2003). Enseñar Ciencias. Barcelona: Graó.

- Capra, F. 1998. La trama de la vida. Barcelona: Editorial Anagrama.

- Mosquera, Diana. 2012. Enseñanza-Aprendizaje del concepto de circulación sanguínea en el ser humano en estudiantes de primaria de zona rural. Tesis para optar el titulo de Magister en enseñanza de ciencias exactas y naturales. Manizales: Universidad Nacional de Colombia.

- Perez, L. (1993). Revisión bibliográfica sobre preconceptos en fisiología de la nutricion humana. Enseñanza de las ciencias. 11 (3). Pp 345-348.

- Nuñez, F \& Banet, E. (1996). Modelos conceptuales sobre las relaciones entre digestión, respiración y circulación. Enseñanza de las ciencias. 14 (3). Pp 261278. 